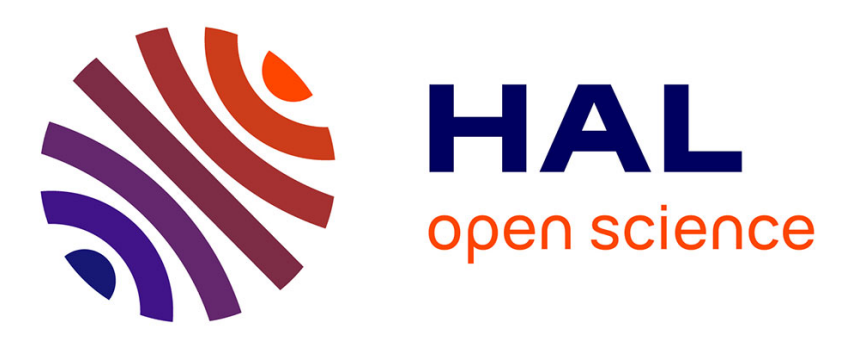

\title{
Transient Complement Inhibition Promotes a Tumor-Specific Immune Response through the Implication of Natural Killer Cells
}

Valérie Janelle, Marie-Pierre Langlois, Esther Tarrab, Pascal Lapierre, Laurent Poliquin, Alain Lamarre

\section{To cite this version:}

Valérie Janelle, Marie-Pierre Langlois, Esther Tarrab, Pascal Lapierre, Laurent Poliquin, et al.. Transient Complement Inhibition Promotes a Tumor-Specific Immune Response through the Implication of Natural Killer Cells. Cancer Immunology Research, 2014, 2 (3), pp.200-206. 10.1158/2326-6066.CIR13-0173 . hal-01199024

\author{
HAL Id: hal-01199024 \\ https://hal.science/hal-01199024
}

Submitted on 14 Sep 2015

HAL is a multi-disciplinary open access archive for the deposit and dissemination of scientific research documents, whether they are published or not. The documents may come from teaching and research institutions in France or abroad, or from public or private research centers.
L'archive ouverte pluridisciplinaire HAL, est destinée au dépôt et à la diffusion de documents scientifiques de niveau recherche, publiés ou non, émanant des établissements d'enseignement et de recherche français ou étrangers, des laboratoires publics ou privés. 


\title{
Transient Complement Inhibition Promotes a Tumor-Specific Immune Response through the Implication of Natural Killer Cells
}

Valérie Janelle 1 , 2 ,

Marie-Pierre Langlois 1 ,

Esther Tarrab1,

Pascal Lapierre1,

Laurent Poliquin2, and

Alain Lamarre 1,

+ Author Affiliations

1. Authors' Affiliations: 'Immunovirology Laboratory, Institut national de la recherche scientifique, INRS-Institut Armand-Frappier, Laval; and ${ }^{2}$ Biomed Research Center, Department of Biology, Université du Québec à Montréal, Montréal, Québec, Canada

1. Corresponding Author:

A. Lamarre, Immunovirology Laboratory, Institut national de la recherche scientifique, INRS-Institut Armand-Frappier, Laval, Québec, Canada H7V 1B7. Phone: 450-687-5010, ext. 4262; Fax: 450-6865501; E-mail: alain.lamarre@iaf.inrs.ca

\begin{abstract}
Although the role of the complement system in cancer development has been studied, its involvement in the development of an antitumoral immune response remains poorly understood. Using cobra venom factor (CVF) to inhibit the complement cascade via C3 molecule exhaustion in immunocompetent mice bearing B16gp33 melanoma tumors, we show that transient inhibition of the complement system allowed for the development of a more robust gp33-specific antitumoral CD8 ${ }^{+} \mathrm{T}$ cell response. This immune response proved to be natural killer (NK) dependent, suggesting an interaction of complement proteins with this cellular subset leading to T lymphocyte activation and enhanced cytotoxic T-cell activity against tumor cells. This study demonstrates for the first time the implication of the complement system in the development of NK-mediated cytotoxic T-cell-dependent antitumoral immune responses. The complement pathway could therefore be a potent therapeutic target to improve NK-dependent antitumoral immune responses in patients with cancer. Cancer Immunol Res; 2(3); 200-6. (02013 AACR.
\end{abstract}

\section{Introduction}

Historically, the complement system has been considered a "complementing" player in the innate immune response that mediated clearance of various pathogens or dead cells and could induce inflammation (1). In cancer, activated complement proteins were primarily described for their role in tumor defense either through complement-dependent cytotoxicity (2) or through antibody-dependent cell-mediated cytotoxicity (ㄱ). However, the complement anaphylatoxins C3a and C5a can increase the activation of phosphatidylinositol 3-kinase, Akt, and mTOR (4); when overexpressed, these proteins are strongly associated with neoplasia, suggesting a more complex role for the complement proteins in tumorigenicity. Furthermore, mice deficient in C3 or C4 show significantly decreased proliferation of TC-1 subcutaneous tumors compared with that in wild-type mice (므), whereas the C5a protein could be involved in CD8 ${ }^{+}$T-cell inhibition ( $\left.\underline{6}\right)$.

Natural killer (NK) cells are large granular lymphocytes that are part of the innate immune system implicated in host defense against tumors and pathogens (ㄱ). NK cells express FcyR, which interacts with immunoglobulin bound to antigen on target cells, leading to NK cell activation (ㅇ) and production of IFN- $\gamma$ and TNF- $\alpha(\underline{9})$. NK cells can also complement CTLs by killing MHC class I-deficient cells that failed to be recognize by T cells (ㄱ). NK cells have been shown to cross talk with dendritic cells; their 
effector functions are stimulated through direct contact with activated dendritic cells (10). However, dendritic cell/NK cell interactions are bidirectional, resulting not only in NK cell activation but also dendritic cell maturation depending on the activation status of both cells (11). Soluble factors such as TNF- $\alpha$ and IFN- $\gamma$, as well as cell-to-cell contact, are required for NK-mediated dendritic cell activation (12).

Little is known about the link between the complement system and NK cells, but evidence suggests that complement receptor CR3, implicated in phagocytosis mediated by complement fragment iC3bopsonized targets (13), is expressed by NK cells and cytotoxic T cells and displayed similar functions as CR3 expressed on phagocytes (14). Given that NK cells can be involved in tumor control and that proteins from the complement pathway may interact with NK cells to suppress or limit their functions, we hypothesized that proteins in the complement pathway may favor tumor growth through the impediment of NK cells. Herein, we found that a transient inhibition of the complement system increased NK cell proportions in the spleen and in the tumor, leading to the induction of an increased antitumoral CTL response.

\section{Materials and Methods}

\section{Cell lines}

Murine B16 and B16gp33 melanoma cells were obtained from Dr. A. Ochsenbein (Department of Clinical Research, University of Bern; Bern, Switzerland). The B16gp33 cells (H2-D $\left.{ }^{b}\right)$ were derived from B16.F10 cells transfected with a DNA minigene encoding the immunodominant $\mathrm{CD} 8^{+} \mathrm{T}$-cell epitope of the lymphocytic choriomeningitis virus (glycoprotein aa 33-41; ref. 15). Cells were grown in Dulbecco's Modified Eagle Medium (Life Technologies) supplemented with $10 \%$ FBS and $200 \mu \mathrm{g} / \mathrm{mL}$ of G418 to select for the retention of the gp33 minigene. No additional test or validation was performed on the cell lines used.

\section{ELISA}

C3 molecule concentration was tested in mice sera at the indicated time points by ELISA. Briefly, blood was harvested and incubated at $37^{\circ} \mathrm{C}$ to coagulate. Samples were centrifuged and the serum fraction was used. Serial dilutions were added onto an immunoglobulin M-coated 96-well plate (5 $\mu \mathrm{g} / \mathrm{mL}$; Sigma-Aldrich). Samples were incubated with mouse anti-C3 coupled to biotin (Cedarlane) followed with streptavidin-horseradish peroxidase (Southern Biotech). A solution of ophenylenediamine dihydrochloride (Sigma-Aldrich) was then used for the detection of C3 molecules. Optical density was measured by spectrophotometry at $490 \mathrm{~nm}$.

\section{In vivo studies}

All procedures were approved by the INRS Institutional Animal Care and Use Committee. To establish subcutaneous tumors, $5 \times 10^{5}$ B16 or B16gp33 cells in $100 \mu \mathrm{L}$ of PBS were injected into the flanks of $\mathrm{C} 57 \mathrm{BI} / 6$ mice. Six days after tumor implantation, mice were injected with cobra venom factor (CVF; 20 $\mu \mathrm{g} /$ mouse; Quidel) intraperitoneally. For NK depletion experiments, $200 \mu \mathrm{g}$ of purified monoclonal antiNK1.1 antibodies from the PK136 hybridoma, kindly provided by Dr. Suzanne Lemieux (Institut national de la recherche scientifique, INRS-Institut Armand-Frappier; Laval, Québec, Canada), were injected intraperitoneally 2 days before decomplementation (Supplementary Fig. S2).

\section{Flow cytometry}

The spleens and tumors were recovered from mice and dissociated in vitro to achieve single-cell suspensions using nylon $100 \mu \mathrm{m}$ cell strainers (BD Falcon). Cells were washed, resuspended in PBS containing $1 \%$ bovine serum albumin and $0.1 \%$ sodium azide (fluorescence-activated cell sorting, FACS, buffer), and incubated with directly conjugated primary antibodies for 30 minutes at $4^{\circ} \mathrm{C}$. Cells were then washed and resuspended in $200 \mu \mathrm{L}$ of FACS buffer containing $1 \%$ paraformaldehyde. Samples were acquired on a FACS Fortessa (BD Biosciences) and analyzed using the Flowjo software (Tree Star). Anti-CD45 phycoerythrin (PE)/CF-594, anti-CD25 allophycocyanin (APC), and anti-NKp46 Alexa 700 were purchased from BD Biosciences. Anti-CD4 APC/Cy7, anti-CD4 FITC, antiCD8 PE/Cy7, anti-CD62L Alexa 700, anti-CD44 PercP/Cy5.5, anti-B220 APC/Cy7, anti-CD11b Pacific Blue, anti-Gr1 PE/Cy5, anti-CD11c FITC, and anti-F4/80 APC were purchased from BioLegend.

\section{Dendritic cell activation}

Single-cell suspensions were prepared from the spleens and draining lymph nodes harvested 9 days after CVF injection. Red blood cells were lysed and the remaining cells were resuspended in FACS 
buffer before CD11C-FITC and CD86-PE labeling (BioLegend). Samples were acquired on a FACS Fortessa (BD Biosciences) and analyzed using the Flowjo software (Tree Star).

\section{NK intracellular staining assay}

For IFN-y intracellular staining, single-cell suspensions were prepared from the spleens and tumors harvested 9 days after CVF injection. Cells were cocultured with YAC-1 cells (provided by Dr. Suzanne Lemieux; at 10:1 effector-target ratio) with or without phorbol 12-myristate 13-acetate (PMA)ionomycin. Cytokine production was measured in the presence of monensin $A(20 \mu \mathrm{g} / \mathrm{mL})$, brefeldin $A$ $(10 \mu \mathrm{g} / \mathrm{mL})$, and anti-CD107a FITC antibody for 5 hours. Cells were stained for surface markers, fixed, and permeabilized for intracellular staining using fixation and permeabilization buffers from BioLegend according to the manufacturer's instructions. IFN-y-PE and CD107a-FITC were obtained from BioLegend.

\section{T lymphocyte intracellular staining assay}

For IFN-y and TNF- $\alpha$ intracellular staining, single-cell suspensions were prepared from the spleens (3 mice/group) 9 days after CVF injection. Cytokine production in response to the tumor antigen was measured by incubation with the gp33 peptide (KAVYNFATC $1 \mu \mathrm{g} / \mathrm{mL}$ ) in the presence of brefeldin A $(10 \mu \mathrm{g} / \mathrm{mL})$ and interleukin (IL)-2 (100 U/mL) for 5 hours. Cells were stained for surface markers then fixed and permeabilized for intracellular staining using fixation and permeabilization buffers as described above. For degranulation assay and granzyme B intracellular staining, cells were incubated with the peptides in the presence of monensin A $(20 \mu \mathrm{g} / \mathrm{mL})$ and anti-CD107a FITC antibody for 5 hours. Cells were stained for surface markers, then fixed and permeabilized for intracellular staining. IFN- $y-P E$ and TNF- $\alpha-A P C$ were obtained from BioLegend and granzyme B eFluor 450 was purchased from eBiosciences.

\section{Statistical analyses}

A Student $t$ test was used for group comparison, and $P$ values of less than 0.05 were considered statistically significant.

\section{Results and Discussion}

\section{CVF-mediated decomplementation slows down early tumor progression and promotes immune cell infiltration}

In recent years, there has been renewed interest in the role of complement in various disease conditions. In cancer, the role of complement is unclear since it has been reported to play a role in both tumor clearance and progression $(2,3)$. Nevertheless, studies have indicated that blocking complement proteins might improve the efficacy of antitumor immunotherapy (16). Some complement activation products, such as the anaphylatoxins $\mathrm{C} 3 \mathrm{a}$ and $\mathrm{C} 5 \mathrm{a}$, are potent proinflammatory mediators, and it is now well recognized that inflammation is able to both promote and exacerbate tumor growth. To establish the role of the complement system in the generation of an antitumoral immune response, transient decomplementation was performed using CVF 6 days following tumor administration, when T-cell priming is expected to occur. CVF is a structural and functional analogue of the C3 molecule, which acts as a C3/C5 convertase. CVF-containing convertases are more stable and resistant to regulatory inhibitors and deplete complement activity by C3 consumption (17). Unlike the complement protein knockout mice (e.g., $\mathrm{C}^{-{ }^{--}}, \mathrm{C}^{-{ }^{--}}$, or $\mathrm{C}^{-/-}$), CVF does not affect the splenic architecture and thus allows a complete and unaltered immune response to be mounted and analyzed (18). Because CVFmediated complement depletion lasts approximately 3 days in treated mice (Supplementary Fig. S1), we hypothesized that this short period of time could affect T-cell priming. CVF-mediated transitory decomplementation soon after tumor implantation efficiently led to a significant slowdown of the growth of B16 melanoma tumors (Fig. 1A). CVF treatment induced a significant increase in the percentage of tumor-infiltrating immune cells 9 days following decomplementation (Fig. 1B). Strikingly, CVF treatment modulated the composition of the infiltrating immune cells; it affected the NK cell proportions, but had little to no effect on the proportions of $\mathrm{CD}^{+}{ }^{+} \mathrm{T}$ cells, regulatory $\mathrm{T}$ cells (Treg), CD8 ${ }^{+} \mathrm{T}$ cells, B cells, neutrophils, macrophages, and dendritic cells (Fig. 1C). 


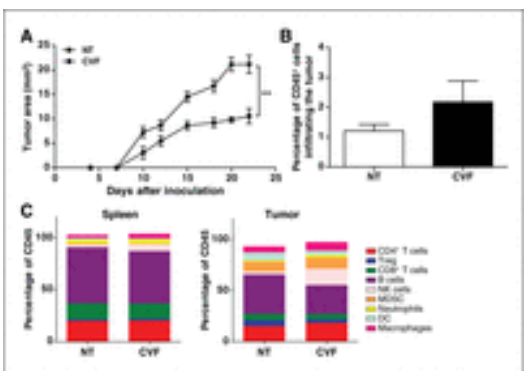

Figure 1.

Effect of CVF treatment on B16 tumor growth and immune cell infiltration. C57BL/6 mice were injected s.c. with B16 (day 0) and CVF was administered by i.p. injection on day 6. A, tumor growth was measured at indicated time points. Data show the mean \pm SEM and are representative of two independent experiments. ${ }^{* *}, P<0.01$. B, 9 days following CVF treatment, tumors and spleens were harvested and total tumor $\mathrm{CD} 45^{+}$cell numbers were determined; $\mathrm{C}$, immune cell subpopulation proportions of $\mathrm{CD}^{+} \mathrm{T}$ cells $\left(\mathrm{CD} 4^{+} \mathrm{CD} 25^{-}\right)$, Tregs $\left(\mathrm{CD} 4^{+} \mathrm{CD} 25^{+}\right), \mathrm{CD}^{+} \mathrm{T}$ cells, B cells $\left(\mathrm{B} 220^{+}\right), \mathrm{NK}^{-}$ $\left(\mathrm{NKp}_{6} 6^{+}\right)$, MDSC $\left(\mathrm{Gr}_{1}{ }^{+} \mathrm{CD} 11 \mathrm{~b}^{+}\right)$, neutrophils $\left(\mathrm{Gr} 1^{+} \mathrm{CD} 11 \mathrm{~b}^{-}\right)$, dendritic cells $\left(\mathrm{Gr}^{-} \mathrm{CD} 11 \mathrm{~b}^{+} \mathrm{CD} 11 \mathrm{c}^{+}\right)$, and macrophages (Gr1-CD11b+F4/80+) were determined. DC, dendritic cells; MDSC, myeloid-derived suppressor cells; NT, not treated.

An important mechanism used by cancer cells to suppress immune responses against tumor antigens is abnormal myelopoiesis and the recruitment of myelomonocytic cells to the tumor microenvironment and peripheral lymphoid organs (19). In concordance with a study conducted by Markiewski and colleagues (므), complement inhibition also significantly decreased the frequency of myeloid-derived suppressor cells (MDSC) in the tumor and the spleen (Fig. 1C and Supplementary Fig. S2). MDSC are a population of intermediately differentiated myeloid cells known to inhibit cancer immunosurveillance and therefore potentiating neoplastic proliferation (2). These cells express high levels of membrane $\mathrm{C} 5 \mathrm{aR}(\underline{5})$. C5a/C5aR binding on MDSC promotes their migration and increases their production of immunosuppressive molecules such as reactive oxygen and nitrogen species, consequently inhibiting cytotoxic $\mathrm{CD}^{+} \mathrm{T}$ lymphocytes and NK cells and stimulating tumorigenic cytokine production and angiogenesis $(\underline{5}, \underline{20})$. MDSC can also be recruited to tumors by the anaphylatoxin C5a deposited in the tumor vasculature (므).

\section{CVF treatment promotes a better NK cell availability}

NK cell proportions were greatly increased following decomplementation both in the tumor (8-fold) and in the spleen (3-fold; Fig. 2A). We next assessed whether decomplementation also modulated NK cell function. Tumoral and splenic NK cells harvested 9 days after CVF treatment were not impaired functionally compared with nontreated animals, as determined by degranulation assay (CD107a) and IFN- $y$ production after exposure to YAC-1 target cells. Furthermore, NK cells retained their ability to respond to ex vivo stimulation (Fig. 2B). Complement proteins are known to induce the production of TGF- $\beta$ (21), which may facilitate angiogenesis, invasion, and metastasis (22), and downregulate the expression of IL-2R $\beta$ as well as the secretion of IFN-y by NK cells (23). Our results suggest that it is unlikely that this pathway is involved here because NK cells harvested from CVF-treated mice are fully functional and respond normally.

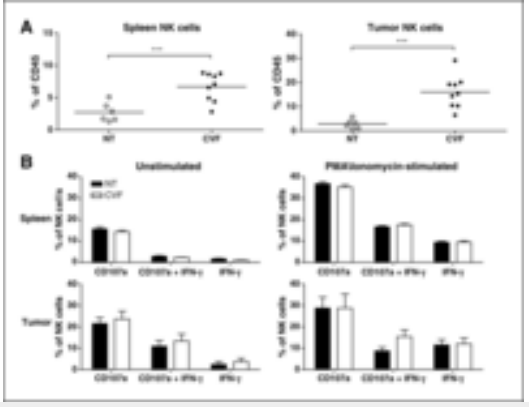

Figure 2.

Decomplementation increases NK cell availability without affecting their effector function. B16gp33bearing mice were treated or not with CVF. On day 9 following treatment, spleens and tumors were harvested. A, percentage of NK cells among CD45 cells. Mean \pm SEM are shown $(n=9)$. 
***, $P<0.001$. B, NK degranulation assay in YAC-1 cocultures (10:1 effector-target ratio) with or without PMA-ionomycin. Data are the mean \pm SEM of three independent experiments $(n=9)$. NT, not treated.

\section{The complement system limits systemic T-cell activation}

It is well recognized that the clearance of apoptotic cells through iC3b opsonization and CR3 phagocytosis can be accompanied by a reduction in the expression of costimulatory molecules and an impaired maturation of dendritic cells (24). The recruitment of large numbers of NK cells may therefore be critical for optimal dendritic cell activation and the subsequent induction of T-cell responses in these low-inflammation conditions. Given that NK cells can cross talk with dendritic cells to mediate their activation, we assessed the activation status of dendritic cells after transient inhibition of the complement system. We did not observe any modulation of the CD86 expression levels in splenic or draining lymph node dendritic cells (Fig. 3A). We then determined whether the enhanced NK cell proportions at the tumor site could lead to an increase in T-cell activation. The majority of $\mathrm{CD}^{+}$and $\mathrm{CD} 8^{+} \mathrm{T}$ cells present in the tumor were of the effector/effector-memory phenotype and their proportions did not change following complement inhibition. However, in the spleen, CVF-mediated depletion of complement led to a reduction in the proportions of naïve T cells associated with increased proportions of effector $\mathrm{CD}^{+}$and $\mathrm{CD} 8^{+} \mathrm{T}$ cells (Fig. 3B). It is therefore likely that, in the absence of complement proteins, functional dendritic cells have access to more tumor antigens due to increased NK-mediated cell lysis allowing for a better antigen presentation to the CD8 $8^{+} \mathrm{T}$ lymphocytes.

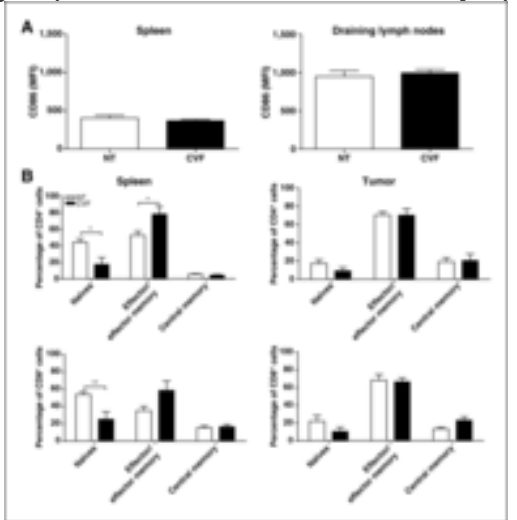

Figure 3.

Increased systemic T-cell activation following CVF treatment. A, spleens and draining lymph nodes were harvested 9 days following CVF treatment, and dendritic cell activation was assessed by CD11C and CD86 staining. Data are the mean \pm SEM of two independent experiments $(n=6)$. B, T-cell activation was assessed by CD62L and CD44 staining on spleens and tumors. Naïve (CD62L $\left.{ }^{+} \mathrm{CD} 44^{-}\right)$, effector/effector memory $\left(\mathrm{CD}_{2} \mathrm{~L}^{-}\right)$, and central memory T cells $\left(\mathrm{CD} 62 \mathrm{~L}^{+} \mathrm{CD} 44^{+}\right)$are shown. Data are the mean \pm SEM of three independent experiments $(n=9) .{ }^{*}, P<0.05 ;{ }^{* *}, P<0.01$. NT, not treated.

The enhancement of tumor-specific CTL responses in decomplemented mice is NK dependent It has been reported that the complement components can interfere with the binding of NK cells to monoclonal antibodies (mAb) used in certain cancer treatments thus limiting NK cell-mediated lysis of antibody-coated target cells (25). This inhibition was shown to be dependent on C3b, likely by its binding to CR3 molecules abrogating signaling via Fcy receptor III (CD16; ref. 25). Furthermore, the depletion of complement has been shown to enhance the survival rates following mAb therapy in a syngeneic mouse model of lymphoma (26).

To ascertain whether the enhanced tumor control in the decomplemented mice was a direct consequence of the increased proportions of NK cells, we depleted NK cells 4 days after tumor implantation. It is important to note that in vivo depletion with anti-NK1.1 antibody is complement mediated, thus required before CVF treatment. We found that in the absence of NK cells, CVF treatment had no effect on tumor implantation and early tumor development, demonstrating that NK cells were necessary for the decreased tumor growth observed following decomplementation (Fig. 4A). 


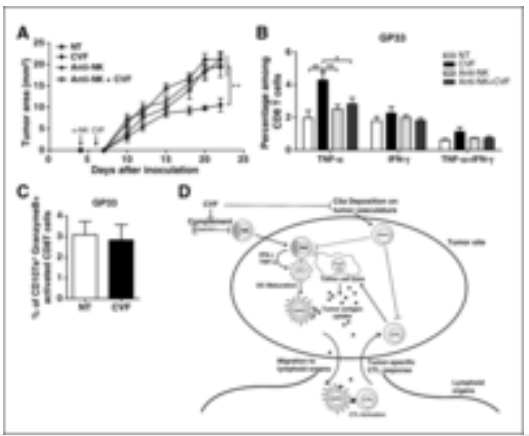

Figure 4.

Influence of NK cells on CVF therapy. A, C57BL/6 mice were injected s.c. with B16 cells $(n=6-8)$, NK depleted or not at day 4 and treated or not with CVF at day 6 . Tumor growth was measured at indicated time points. Data show the mean \pm SEM and are representative of two independent experiments. ${ }^{*}, P<0.05 ;{ }^{* *}, P<0.01$. B, C57BL/6 mice were injected s.c. with B16gp33 cells, NK depleted or not at day 4 and treated or not with CVF at day 6 . Nine days following CVF treatment, spleens were harvested and an ex vivo stimulation with the gp33 (KAVYNFATC) peptide was performed to analyze cytokine secretion and C, degranulation. Data are the mean \pm SEM of two independent experiments $(n=6)$. D, the proposed model for the influence of complement proteins and NK cells in the B16 melanoma model. Inhibition of the complement system by CVF allows for increased NK cell availability in tumor tissues. This expanded proportion of NK cells leads to increased tumor cell lysis and antigen release, thus facilitating the activation of tumor-specific CD8 ${ }^{+} \mathrm{T}$ lymphocytes. Inhibition of complement proteins also acts on MDSC and limits the immunosuppressive tumor microenvironment, leading to increased CTL activation. iDC, immature dendritic cell; mDC, mature dendritic cell; NT, not treated.

Considering that T-cell activation in the spleen is also increased in the absence of complement, we determined the effect of decomplementation on the induction of tumor-specific CTLs. As assessed by intracellular cytokine staining of gp33 peptide-stimulated T cells from decomplemented and control B16gp33-bearing mice, we found that complement inhibition led to higher levels of tumor-specific (gp33) T cells secreting mainly TNF- $\alpha$ (Fig. 4B). Accordingly, the enhancement of gp33-specific CTL induction by CVF was also abolished in the absence NK cells, indicating that the effect of decomplementation on early tumor growth is largely mediated by CTLs (Fig. 4B). However, CVFmediated decomplementation did not lead to superior levels of gp33-specific cytotoxicity as assessed by CD107a expression, a marker of recent degranulation (Fig. 4C). Thus, using the B16 melanoma model, we demonstrate that CVF treatment limits early tumor growth and promotes enhanced availability of the splenic and tumoral NK cells.

Therefore, our study has identified a previously unrecognized function for complement in tumor biology. We have shown that complement components and NK cells interact and influence tumor growth by limiting tumor-specific CTL induction. Factors from the complement system may restrict NK cell availability in tumor tissue in which NK cells would normally participate in the induction of a tumorspecific CTL response via, among other things, tumor cell lysis. Furthermore, complement proteins could also promote infiltration of the tumor by MDSCs that could then suppress lymphocyte function (Fig. 4D).

Many drugs targeting complement proteins are currently in clinical trials, and few side effects have been reported $(1,27)$. CVF has been used in laboratory animals for the treatment of various diseases. One of the limits of this treatment alternative in the clinic is the high immunogenicity of this compound (28). However, a human C3 hybrid, containing crucial regions of the CVF $\beta$ chain (so-called humanized CVF or HC3-1496), has been created for this purpose (29). It will be of great interest to test other complement inhibitors currently in development in various solid tumor models to determine whether sustained treatment will favor the induction of an improved tumor-specific immune response and promote $\mathrm{CD}^{+} \mathrm{T}$-cell expansion. Thus, a better understanding of the link between the complement proteins and NK cells in the developing tumors could lead to the design of new therapeutic strategies against cancer.

\section{Disclosure of Potential Conflicts of Interest}


No potential conflicts of interest were disclosed.

\section{Authors' Contributions}

Conception and design: V. Janelle, A. Lamarre

Development of methodology: V. Janelle, M.-P. Langlois, E. Tarrab, A. Lamarre

Acquisition of data (provided animals, acquired and managed patients, provided facilities, etc.): V. Janelle, M.-P. Langlois

Analysis and interpretation of data (e.g., statistical analysis, biostatistics, computational analysis): V. Janelle, M.-P. Langlois, P. Lapierre, A. Lamarre

Writing, review, and/or revision of the manuscript: V. Janelle, P. Lapierre, L. Poliquin, A. Lamarre

Administrative, technical, or material support (i.e., reporting or organizing data, constructing databases): V. Janelle, M.-P. Langlois

Study supervision: V. Janelle, E. Tarrab, L. Poliquin, A. Lamarre

\section{Grant Support}

This work was supported by the Jeanne and J.-Louis Lévesque Chair in Immunovirology (to A. Lamarre) from the J.-Louis Lévesque Foundation. V. Janelle and M.-P. Langlois acknowledge studentship support from the Fondation Armand-Frappier. P. Lapierre holds a Fonds de recherche du Québec-Santé (FRQS) postdoctoral award and a Thomas F. Nealon, III Postdoctoral Research Fellowship from the American Liver foundation.

\section{Acknowledgments}

The authors thank Simon Janelle for his help on the schematic illustration of the proposed model.

\section{Footnotes}

- Note: Supplementary data for this article are available at Cancer Immunology Research Online (http://cancerimmunolres.aacrjournals.org/).

- Received October 3, 2013.

- Revision received October 28, 2013.

- Accepted November 21, 2013. 